\title{
Hunting osteoporosis susceptibility genes: bigger is better but diverse is also welcome
}

\author{
Fernando Rivadeneira
}

Received: 8 September 2012/ Accepted: 12 September 2012/Published online: 22 September 2012

(C) Springer Science+Business Media New York 2012

Osteoporosis causes more than 8.9 million fractures annually worldwide with the lifetime risk for a wrist, hip, or vertebral fracture estimated to be in the order of 30-40\% in developed countries [1]. Osteoporosis is not only a major cause of fractures but also ranks high among diseases causing people to become bedridden with high morbidity, mortality, and costs to the individual and the society. Osteoporosis has been operationally defined on the basis of the bone mineral density (BMD) assessment. Nevertheless, the BMD measurement alone is not optimal for the detection of individuals at high risk of fracture. Even though the risk of fracture is very high when BMD is low (high specificity), risk is not negligible when BMD is normal (low sensitivity). This has to do with the multifactorial etiology of osteoporosis and its associated fractures. Multiple underlying factors influence the risk of disease, including age, sex, menopausal status, diet, physical activity, smoking, coexisting diseases, and pharmacologic treatments. Family history of fracture is another strong confirmed risk factor which has brought great expectation of what the assessment of genetic factors can bring to understand the underlying biology, improve risk prediction, and develop novel disease interventions (treatment).

As in other human complex diseases, the studies on the genetics of osteoporosis have now begun a new era of discoveries with the advent of the so-called genome-wide association studies (GWAS) [2]. GWAS were made possible by the knowledge provided by the HapMap project (on the organization of common variation in the genome) and the introduction of high throughput genotyping

F. Rivadeneira $(\square)$

Departments of Internal Medicine and Epidemiology, Erasmus

MC, P.O. Box 2040 Ee5-79, 3000 CA Rotterdam, The

Netherlands

e-mail: f.rivadeneira@erasmusmc.nl technology (allowing assessing hundreds of thousands of DNA polymorphisms in adequately powered settings, including tenths of thousands of individuals). Before the GWAS era, the literature about the genetics of osteoporosis and fracture had been confined to a very large number of "genome-wide linkage" and "candidate gene association" studies. In retrospect, and with few exceptions, the majority were small, inadequately-powered studies generating controversial and frequently non-reproducible reports [3] on variants in about 150 candidate gene regions for osteoporosis according to HuGeNet [4]. Even though, a few polymorphisms were indeed identified as being associated with either BMD or fracture (such as for LRP5 [5] associated at $P<5 \times 10^{-8}$, the current standard for declaring genome-wide significance). Candidate gene efforts were then restricted to a number of known ill-selected polymorphisms and did not interrogate the genetic contribution to osteoporosis in a robust and hypothesis-free way, as is now possible by means of the GWAS approach.

In this issue of the Journal, Deng et al. has scrutinized 18 SNPs for association with BMD and fracture risk in a relatively small population $(n=1,012)$ of Chinese Han women [6]. The markers arise from 16 BMD loci identified by the first large scale GWAS meta-analysis of the Genetics Factors of Osteoporosis (GEFOS) consortium [7]. Such independent validation effort is particularly welcome in the field of genetics of osteoporosis considering it is drawn in individuals of a non-European background. Further, they attempted to evaluate the relation with fracture risk which will always be one step further toward fulfilling the expectations on clinical translation described above.

The authors encounter evidence for an association with BMD in SNPs from six loci, including 1p31.3 (containing WLS formerly known as GPR177), 3p22.1 (CTNNB1), 
5q14.3 (MEF2C), 7q21.3 (FLJ42280), $8 \mathrm{q} 24.12$ (TNFRSF11B), and 11p15.1 (SOX6). In addition, one SNP in the 2p16.2 locus mapping to SPTBN1 was associated with 1.4 [95 \% CI (1.016-1.900)] increased risk for osteoporotic fracture. Altogether, these findings corroborate the involvement of factors acting through important pathways known to play an important role in bone biology, like mesenchymal cell differentiation, OPG-RANK-RANKL, TGF- $\beta$, and Wnt signaling.

As encouraging this, cross-ethnic replication can be perceived; several aspects require careful interpretation. As is frequently the case for complex traits and common diseases hundreds (if not thousands) of variants with weak (but genuine) effects will be underlying their genetic architecture [8]. Allocating a gene to a genetic signal is based on physical distance and current knowledge of biology, neither of which suffices to unequivocally assign the gene to the observed SNP effect. Therefore, even though the circumstantial evidence for biologic candidacy may be strong, such assignments should be interpreted with caution until conclusive evidence becomes available. This will also vary from region to region depending on the relative location of the associated SNPs, the linkage disequilibrium (LD) properties between the markers, the patterns of recombination rate, and the number of genes underlying the signal. Considering the limited power of the replication setting in Deng et al., excluding the involvement of the non-associated variants in Asian populations will be premature and likely a misjudgment. Different LD regional structure between populations can result in additional hampering of the power for association. The top associated marker in populations of European background, in relatively high $\mathrm{LD}$ with the real underlying variant(s) driving the associations, may withhold low(er) correlation in Asian populations. This will result in further power limitations and higher likelihood of false negative associations been drawn for a given locus. Such is also the case, when drawing conclusions on potential site specificity of the observed effects, where also the absence of evidence (of association) will not constitute the evidence of absence. Given the relatively high phenotypic correlation between BMD measured at the lumbar spine and the total hip $(r 2-0.70)$, it is expected that most loci will show association at both skeletal sites. Larger collaborative studies in Asian populations are required to address these contentions.

The recently published second effort on BMD of the GEFOS consortium has brought together about 80,000 individuals with DXA scans from 50 studies across Europe, North America, East Asia and Australia [9]. This study constitutes the largest genetic study in osteoporosis performed to date and has now brought the number of identified BMD loci to 56, including all 16 loci assessed by
Deng et al. in this issue. Fourteen of the 56 BMD loci were associated with any type of fracture, examined in more than 30,000 cases and 100,000 controls. As also found by Deng et al., variants in the $2 \mathrm{p} 16.2$ locus mapping to SPTBN1 were associated with fracture, yet at genome-wide significant level. Further, within GEFOS, all effect sizes were under $10 \%$ increased risk which represents a more precise (and expected) risk estimate, than the $40 \%$ reported by Deng et al. in this issue.

We can expect that applying the GWAS approach in an ever-expanding number of individuals will continue allowing the identification of hundreds of common variants underlying the risk of osteoporosis and fracture. This approach will be complemented by the implementation of sequencing technologies and functional follow-up of the identified loci and their pathways. All together, these discoveries will help understand the genetic underpinnings of a complex disease like osteoporosis, setting the roadmap to pinpoint drug targets and provide leads for the development of improved therapies and preventive measures.

\section{References}

1. C. Cooper, The crippling consequences of fractures and their impact on quality of life. Am. J. Med. 103(2A), 12S-17S (1997); discussion $17 \mathrm{~S}-19 \mathrm{~S}$

2. L.A. Hindorff et al., Potential etiologic and functional implications of genome-wide association loci for human diseases and traits. Proc. Natl. Acad. Sci. USA 106(23), 9362-9367 (2009)

3. J.P. Ioannidis, Why most published research findings are false. PLoS Med. 2(8), e124 (2005)

4. J. Little, S. Hawken, On track? Using the human genome epidemiology roadmap. Public Health Genomics 13(4), 256-266 (2010)

5. J.B. van Meurs et al., Large-scale analysis of association between LRP5 and LRP6 variants and osteoporosis. JAMA 299(11), 1277-1290 (2008)

6. Y. Deng et al., The influence of the genetic and non-genetic factors on bone mineral density and osteoporotic fractures in Chinese women. Endocrine (2012). doi:10.1007/s12020-0129726-8

7. F. Rivadeneira et al., Twenty bone-mineral-density loci identified by large-scale meta-analysis of genome-wide association studies. Nat. Genet. 41(11), 1199-1206 (2009)

8. H. Allen Lango et al., Hundreds of variants clustered in genomic loci and biological pathways affect human height. Nature 467(7317), 832-838 (2010)

9. K. Estrada et al., Genome-wide meta-analysis identifies 56 bone mineral density loci and reveals 14 loci associated with risk of fracture. Nat. Genet. 44(5), 491-501 (2012)

\section{Websites}

GWS Catalogue: http://www.genome.gov/gwastudies GEFOS consortium: http://www.gefos.org/

HUgeNet: http://www.hugenavigator.net/ 